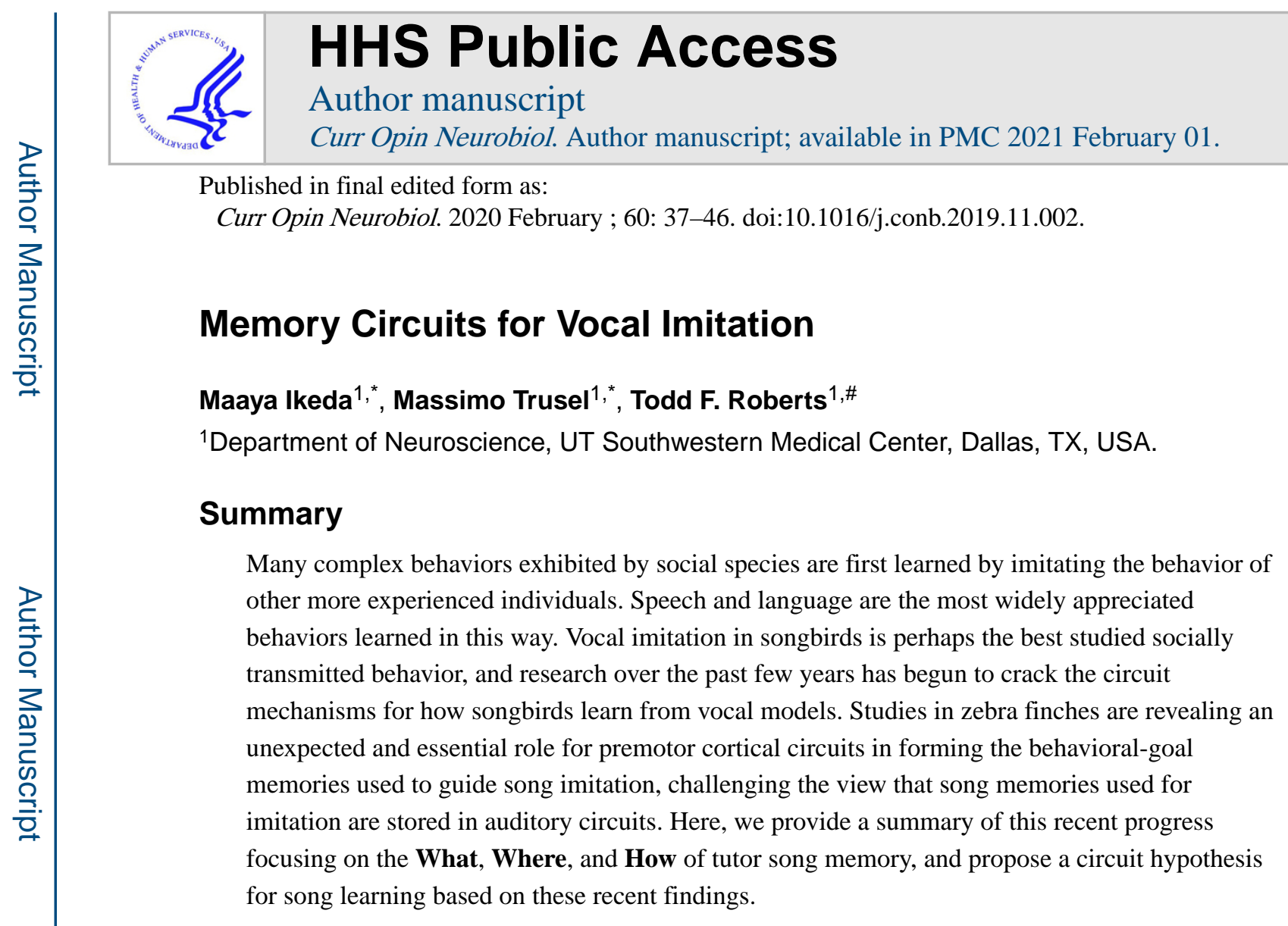

\title{
Vocal Imitation
}

People and other animals learn many of their complex and socially oriented behaviors by imitating more experienced individuals in their environment. Prerequisites to this form of learning are the ability to encode memories of social models, and the facility to use those memories to guide changes in motor performances (Bolhuis and Gahr, 2006; Doupe and Kuhl, 1999; Roberts and Mooney, 2013). Speech and language are among the most remarkable examples of this form of learning. The broad importance of imitative learning can be appreciated when one considers that developmental delays or disruptions in speech and language are key early indicators of a variety of neurodevelopmental disruptions and can be diagnostic of autism spectrum disorders (Mody and Belliveau, 2013; Sperdin and Schaer, 2016; Tager-Flusberg et al., 2005).

Using model systems to examine the circuit mechanisms for imitative learning is therefore essential to understanding the acquisition of social skills and how neurodevelopmental disorders derail this important learning process (Brainard and Doupe, 2013). However, studying imitation in the laboratory is complicated by its social nature and the difficulties in isolating discrete and quantifiable imitated behaviors. For example, rodents and non-human

\footnotetext{
*Correspondence and requests for materials should be addressed to T.F.R. (todd.roberts@utsouthwestern.edu). *these authors contributed equally

Author Information: The authors declare no competing financial interests.

Publisher's Disclaimer: This is a PDF file of an unedited manuscript that has been accepted for publication. As a service to our customers we are providing this early version of the manuscript. The manuscript will undergo copyediting, typesetting, and review of the resulting proof before it is published in its final form. Please note that during the production process errors may be discovered which could affect the content, and all legal disclaimers that apply to the journal pertain.
} 
primates do not appear to imitate skilled communicative behaviors like vocalizations, but rather communicate using innately specified vocal repertoires (Konopka and Roberts, 2016; Portfors and Perkel, 2014). Songbirds, like people, require experience from vocal models to guide learning of their song during development (Doupe and Kuhl, 1999). The study of birdsong learning has therefore provided the principal laboratory model for understanding 1) how neural circuits organize to encode memories from social models that are used to guide imitation, 2) how sensory feedback is integrated to compare the present performance to the vocal target, and 3) how the brain engages in error correction during motor imitation for lifelong maintenance of these imitated vocalizations. Zebra finches imitate the song of their father or other adult male bird during a development critical period and have specialized auditory and motor circuitry for learning and producing song (Figure 1). Studies in zebra finches have begun to isolate the synaptic pathways involved in forming memories of adult vocal models used to guide song imitation, a memory we refer to as a behavioral-goal memory.

Several recent reviews have drawn on the large immediate-early gene (IEG) circuit mapping literature to propose that auditory cortical circuits store tutor song memories (Bolhuis and Gahr, 2006; Bolhuis and Moorman, 2015; Moorman et al., 2011). We focus our review on recent functional and electrophysiological studies which have started to directly test this now widely held view for song memory. Crucially, by distinguishing between circuits involved in forming perceptual memories for song from circuits involved in forming behavioral-goal memories used to guide imitation, this recent literature indicates an essential role of song motor circuits, not auditory circuits, in the initial encoding of behavioral-goal memories.

\section{What? Perceptual Memories for Birdsong and Behavioral-Goal Memories}

For social species, recognizing the vocalizations of caregivers and other individuals within the social group is crucial for survival. Experience-dependent tuning of receptive fields in the auditory cortex (AC) is a likely mechanism for developing selectivity for conspecific vocalizations (Kuhl et al., 1992; Poremba et al., 2013; Werker and Tees, 1992; Woolley et al., 2005). Like the mammalian AC, the avian AC contains neurons that receive input from the auditory thalamus and secondary circuits important for higher-order processing of auditory information. Neurons in secondary regions, including NCM and CM (see legend of figure 1 for abbreviations), exhibit selectivity for spectral and temporal aspects of conspecific vocalizations. In a recent study using simultaneous recordings from multiple regions of the AC, Moore \& Woolley (2019) showed that auditory selectivity of species' typical vocalizations emerges in secondary portions of the songbird AC. When birds are reared by tutors of another species, neurons in secondary regions become more responsive to songs and sounds that are spectrally and temporally common in this other species, indicating the role of experience and the $\mathrm{AC}$ in recognition of conspecific songs or socially relevant sounds (Moore and Woolley, 2019).

The original "acquired auditory template" hypothesis for tutor song memory implicates auditory experience of the tutor as a necessary step in the formation of a tutor song memory (Konishi, 1965; Marler, 1970). This "auditory template" is a central component of all song learning models. In its most basic form, the auditory template functions as a memory of the 
tutor song. This memory serves as an internal benchmark used to evaluate sensory (auditory) feedback of vocal performances. Errors in performance are calculated by comparing auditory feedback with this auditory template and detected errors are then used to guide changes in behavior (Fee and Goldberg, 2011; Keller and Hahnloser, 2009; Mandelblat-Cerf et al., 2014; Mooney, 2009).

Given the role of the AC in coding for conspecific vocalizations, it is reasonable to guess that specialized circuits or neurons in the AC encode perceptual memories of the tutor song that may also serve as the auditory template. Indeed, several lines of evidence, including a large body of IEG literature, have implicated NCM as the site of the auditory template (see Bolhuis and Moorman, 2015 for review of this literature). However, interpreting this literature can be tricky. Songbirds imitate the song of their tutor, resulting in strong correlations in the acoustic properties of the bird's own song (BOS) and the song of their tutor. Studies have not consistently examined if IEG expression is driven by playback of tutor song independent of BOS, leaving it unclear whether IEG responses are tutor specific or reflect corollary responses from BOS-tuned neurons. More recently, electrophysiological recordings from NCM in juvenile birds have identified a small subset of neurons that are highly selective to playback of the tutor song but not BOS (Yanagihara and YazakiSugiyama, 2016). The functional significance of these neurons in guiding song imitation is currently not known and their sparse distribution throughout NCM is likely to challenge the ability to selectively manipulate or lesion these neurons while sparing other portions of NCM. Other possibilities are (i) that perceptual memories of the tutor song, identified by auditory playback experiments, are completely independent of memories used to guide imitation, (ii) that memories used to guide imitation reside in regions downstream of the AC, and/or (iii) that memories of the tutor song are state-dependent and only accessible while the bird is singing, rather than passively listening to songs (Roberts et al., 2017; Roberts and Mooney, 2013).

Although the acquired auditory template hypothesis is suggestive of an auditory representation, it does not postulate that the neural representation of this memory would be engraved solely in auditory circuits. The AC sends afferents to two sensorimotor structures, NIf and $A v$, which in turn provide input to the song system via the song premotor nucleus HVC (Akutagawa and Konishi, 2010; Bauer et al., 2008; Cardin et al., 2005; Cardin and Schmidt, 2004; Coleman and Mooney, 2004; Janata and Margoliash, 1999; Lewandowski et al., 2013; McCasland, 1987). Auditory playback experiments in juveniles have identified tutor responsive neurons in several portions of the song motor system and peripheral manipulations that disrupt singing have shown that these responses can be independent of BOS-tuned responses (Adret et al., 2012; Nick and Konishi, 2005b; Prather et al., 2010; Solis and Doupe, 1999; Solis and Doupe, 2000; Vallentin et al., 2016; Volman, 1993).

During development initially weaker tutor responses largely give way to responses that are selective to the birds own song (Nick and Konishi, 2005a; Solis and Doupe, 1999; Vallentin et al., 2016), a finding that has been used to argue that song motor circuits only represent a template for song production and not a memory of the tutor song (Bolhuis and Moorman, 2015). However, this argument stems from the assumption that the 'auditory template' is stored as a perceptual memory that can be mapped by playback of the tutor song to waking 
or anesthetized birds. While this assumption may be historically embedded in the idea of an acquired auditory template, there is no a priori justification to suggest that information used to guide imitation can be accessed by auditory playback experiments. It is possible that perceptual memories of the tutor song and the memory used to guide imitation are independently represented in the brain and/or that memories that guide vocal imitation are only accessible during singing. As discussed in the following sections, several lines of evidence indicate that tutoring experience is rapidly encoded in the song system. We suggest that in its most common use, 'auditory template' fails to capture the potential distinction between perceptual memories and memories that guide imitation. Indeed, it is common for 'auditory template' to be used as a descriptor of a perceptual memory of a tutor song without addressing if this memory functions to guide vocal imitation. To avoid this confusion, we use 'behavioral-goal memory' to specify the memory that guides vocal imitation (Zhao et al., 2019).

\section{Where? Functional Dissection of Behavioral-Goal Memories}

IEG mapping studies and electrophysiological findings have highlighted the potential role of the AC regions NCM and CM for encoding tutor's song memory. Additionally, transient inhibition of mitogen-activated protein kinase/ERK kinase (MEK) in primary or secondary regions of the zebra finch $\mathrm{AC}$, including $\mathrm{NCM}$, are reported to disrupt song learning (London and Clayton, 2008). Nonetheless, lesion studies indicate that NCM is unlikely to be important for encoding behavioral-goal memories. Large bilateral lesions of NCM do not impair juvenile zebra finch's ability to learn how to imitate the song of a tutor (Canopoli et al., 2016), arguing that the memory used to guide imitation is not located in NCM or, at a minimum, not uniquely located in NCM. Moreover, bilateral lesions of NCM prior to tutoring of young birds also fail to disrupt acquisition of a behavioral-goal memory, seen by the subsequent normal imitation of the tutors' song (Canopoli et al., 2017). Although lesion studies can be difficult to interpret and further functional experiments will be required, it appears that NCM is not necessary for initial encoding of a behavioral-goal memory or for the use of this memory to guide song imitation. A parsimonious interpretation of the available data is that NCM is involved in tutor song recognition or perception, a process that could be independent of encoding a behavioral-goal memory used for song imitation.

$\mathrm{CM}$ and its subnucleus Av have also been implicated in encoding behavioral-goal memories. However, the functional significance of these regions has not been well tested. Av is reciprocally connected with $\mathrm{HVC}$ and NIf, and its neurons project to the intermediate arcopallium (AIV), which in turn projects to the ventral tegmental area (VTA) (Akutagawa and Konishi, 2010; Mandelblat-Cerf et al., 2014; Roberts et al., 2017; Zhao et al., 2019). Electrophysiological recordings from Area X-projecting VTA dopaminergic neurons indicate that they encode reward prediction errors for song performance (Gadagkar et al., 2016), and singing-contingent optogenetic manipulations indicate that VTA inputs to Area X are sufficient to positively and negatively reinforce changes in song (Hisey et al., 2018; Xiao et al., 2018). Neurophysiological correlates of singing-related error signals have been observed in AIV and in portions of CM overlapping with Av (Keller and Hahnloser, 2009; Mandelblat-Cerf et al., 2014). Av receives inputs from the song premotor region HVC, and genetic lesions of Av-projecting HVC neurons or direct lesions of AIV prevent juvenile 
birds from copying the song of their tutor (Mandelblat-Cerf et al., 2014; Roberts et al., 2017). Therefore, it is likely that the behavioral-goal memory used to evaluate auditory feedback and calculate error signals is stored, at least in part, upstream of Av and AIV.

In the last decade, several lines of evidence have pointed to a role for $\mathrm{HVC}$ in the encoding of behavioral-goal memories. HVC synapses undergo stabilization and enlargement immediately following experience with a song tutor, and the levels of spine turnover measured prior to tutoring are predictive of a bird's subsequent ability to learn and match the tutor's song (Roberts et al., 2010). Tutoring experience results in the rapid emergence of bursting activity and auditory selectivity to playback of the tutor song (Roberts et al., 2010; Shank and Margoliash, 2009; Tanaka et al., 2018; Vallentin et al., 2016). In addition, tutor responsive neurons in $\mathrm{HVC}$ have been identified even in young birds prior to the onset of singing (Adret et al., 2012). These correlative findings strongly implicate HVC in the initial encoding of behavioral-goal memories.

More causal evidence of HVC's involvement in memory storage has come from the disruption or facilitation of tutor song memorization by optogenetic and pharmacological manipulations in HVC. Infusion of the NMDA receptor antagonist (2R)-amino-5phosphonovaleric acid (APV) in HVC during tutoring blocks encoding of a behavior-goal memory (Roberts et al., 2012). Closed-loop tutor-song contingent optogenetic manipulation of HVC activity also blocks the encoding of a behavioral-goal memory in juvenile birds (Roberts et al., 2012). In contrast, stimulation of midbrain (A11) dopaminergic fibers within HVC facilitates the memorization and increased imitation of song that is passively played back from a speaker, a stimulus that zebra finches typically fail to imitate (Tanaka et al., 2018). Moreover, pharmacological block of dopaminergic signaling in HVC, but not CM or Area X, disrupts the ability of young birds to encode tutor song memories (Tanaka et al., 2018). Together these findings suggest that tutoring-triggered reorganization of circuits within $\mathrm{HVC}$ is instrumental to the encoding of a behavioral-goal memory, and that dopaminergic inputs to HVC facilitate learning from social models.

Significant progress has also been made in identifying the circuits that relay tutor-song information to HVC during learning. As mentioned above, tutor experience results in rapid reconfiguration of spontaneous activity in the song system. In adult birds, this bursting activity in HVC is driven by inputs from NIf (Cardin et al., 2005; Coleman and Mooney, 2004; Hahnloser and Fee, 2007; Roy and Mooney, 2009). Chemical or genetic lesions of NIf prior to tutoring block encoding of behavioral-goal memories (Roberts et al., 2012; Zhao et al., 2019). Zhao and colleagues have recently shown that NIf contains two largely separate classes of projection neurons, either projecting to HVC or to Av, and genetic lesions of only the HVC projection neurons in young birds blocks their ability to encode a memory of a tutor song (Zhao et al., 2019). However, if lesions are conducted in juvenile birds after they have had experience with a tutor, birds could still learn to imitate the previously memorized song (Zhao et al., 2019). This suggests that the tutor song memory is ultimately encoded downstream of NIf to HVC synapses and that NIf-HVC neurons play a privileged role in relaying tutor song information to HVC. 
Consistent with the idea that the NIf-HVC pathway carries tutor-song information to circuits encoding song memories, Zhao et al also showed that behavioral-goal memories can be artificially implanted by optogenetically stimulating NIf axon terminals in HVC of juvenile birds (Zhao et al., 2019). This opto-tutoring paradigm was used to implant simple behavioral-goal memories for song timing. Normal zebra finch song syllables are $\sim 100 \mathrm{~ms}$ in duration. Either $50 \mathrm{~ms}$ or $300 \mathrm{~ms}$ light pulses were delivered in a pattern mimicking natural tutoring to socially isolated juvenile birds when they were alone in a cage and not singing. In the case of birds opto-tutored with 50ms light pulses, this resulted in birds learning significantly shorter song elements than those produced by normal birds or by isolate birds. By contrast, opto-tutoring with 300ms pulses of light resulted in the birds learning song elements that were significantly longer than isolate or normal birds. Consistent with the idea of implanting artificial behavioral-goal memories for a song, birds showed normal song learning trajectories and only learned to approximate the artificial song memories following extensive practice and refinement of vocal patterns. Opto-tutored birds also used these artificially-shaped songs as adults in everyday singing, and in female-directed singing, strengthening the idea that opto-tutoring accesses the same brain pathways, and perhaps physiological mechanisms, engaged while learning via social interactions with a song tutor (Zhao et al., 2019).

When taken together, the current evidence suggests that auditory circuits, like NCM, are not essential for the long-term storage or initial encoding of behavioral-goal memories used for vocal imitation. Instead, sensorimotor synapses between NIf and HVC are essential for the bird's ability to initially encode behavioral-goal memories. Whether it is possible to identify a single locus for the long-term storage of the behavioral-goal memory is still unclear. It is clear however that memories are stored downstream of NIf synapses on HVC neurons. Therefore, memories could be stored in other synaptic connections in HVC or in a more distributed manner in circuits downstream of HVC. It's possible, and perhaps likely, that behavioral-goal memories exist in a form that does not cleanly conform to perceptual memories, which can be mapped using auditory playback experiments. In this scenario, the acquired auditory template could exist not as an auditory trace, but as a state-dependent memory that is only accessible during singing. One idea is that corollary discharge signals associated with descending motor commands could recall these 'memories' on a momentby-moment basis, resulting in activation of activity patterns that could then be directly compared to auditory feedback via connections from the motor system back into the AC. We expand on this idea below.

\section{How? Experience-dependent Shaping of Activity in Vocal Motor Circuitry}

The synaptic mechanisms used to encode tutor song memories are still poorly understood, as are the neuronal cell types in HVC that initially encode information from NIf or from the A11 dopaminergic neurons. It is likely that NMDA-dependent mechanisms play a significant role in learning from tutoring experience (Roberts et al., 2012), but synaptic plasticity may not be the only game in town. Ross and colleagues recently found that tutoring experience results in cell-type specific changes in the intrinsic physiological properties of HVC neurons projecting to RA and those projecting to Area X (Ross et al., 2019). An important future 
direction will be to examine the interaction between experience-dependent synaptic and intrinsic plasticity in $\mathrm{HVC}$ and other areas shaped by tutoring experience.

One way in which these forms of plasticity may interact is in the emergence of bursting activity following tutoring experiences (Roberts et al., 2010; Shank and Margoliash, 2009; Tanaka et al., 2018). At the circuit level, it is clear that forming a behavioral-goal memory strengthens synaptic connections between NIf and HVC and that it reshapes neuronal activity in NIf, HVC and RA. Although the overall activity in HVC does not change, high frequency burst firing rapidly increases following encoding of behavioral-goal memories and this bursting depends on inputs from NIf (Roberts et al., 2010; Tanaka et al., 2018). Bursting activity in the song system is a widely appreciated phenomenon and it is routinely used to identify the song nuclei from surrounding brain regions. The behavioral function of this spontaneous bursting activity is not known, but the fact that it arises precisely following memory formation raises curiosity as to its role in behavioral-goal memories. Indeed, Shank and Margoliash examined the inter-spike interval (ISI) in high frequency burst firing in RA following tutoring experience and remarkably found that ISI distributions across birds clustered based on the song of their tutor (Shank and Margoliash, 2009). Although this latter study awaits independent confirmation, together with the now numerous reports of tutoringdependent changes in the song system, it lays a potential roadmap for examining how behavioral-goal memories could be imprinted in the bursting patterns that flow through vocal motor circuits (Roberts et al., 2012; Roberts et al., 2010; Ross et al., 2019; Shank and Margoliash, 2009; Tanaka et al., 2018; Vallentin et al., 2016; Zhao et al., 2019).

\section{Song Imitation without NCM?}

Our review of the literature indicates that behavioral-goal memories are initially encoded or at least must relay through HVC. So where does it go from there and what is the circuit architecture that allows the behavioral-goal memory to be compared with auditory feedback? Recently proposed models of song learning implicate the $\mathrm{AC}$ as the final source for behavioral-goal memories and suggest that song evaluation occurs in Av and AIV (Chen et al., 2019; Kearney et al., 2019; Mackevicius and Fee, 2018; Roberts et al., 2017). These regions are hypothesized to compare ascending auditory feedback to 1) an auditory template relayed from NCM projections to Av and AIV and 2) a corollary discharge of the descending song motor command relayed through HVC's projection onto Av. During encoding of behavioral-goal memories it is possible that tutor-song information passes through HVC and flows directly back into the auditory system via Av, thereby allowing the formation of a long-term memory. Lesion studies reviewed here indicate that NCM is not where behavioral-goal memories are ultimately stored (Canopoli et al., 2016, 2017); however, NCM is only one portion of a large AC and it's possible that behavioral-goal memories are distributed throughout the entirety of the AC or selectively encoded in portions of AC outside of NCM.

Unlike lesions of NCM, genetic lesions of HVC-Av neurons in juvenile birds are sufficient to disrupt song imitation (Roberts et al., 2017). HVC-Av neurons are proposed to carry a copy of the descending vocal-motor command to the auditory system and Roberts et al proposed that the HVC-Av projections carry a corollary discharge signal that reactivates the 
behavioral-goal memory in the AC and temporally registers it with auditory feedback as birds sing. Given the dearth of functional data indicating a strong role for the AC in encoding behavioral-goal memories, it is interesting to speculate if a simpler model could account for song learning.

For example, what if HVC neurons encode aspects of the behavioral-goal memory as a statedependent memory recalled on a moment-by-moment basis during singing. In this scenario, the corollary discharge signal comes from HVC-RA neurons axonal collaterals synapsing on other neurons in HVC, including interneurons and HVC-Av neurons. This excitatory input during singing temporally aligns or "calls-up" the correct aspects of the behavioral-goal memory - a synaptic mapping learned during auditory experience with a song tutor and potentially strengthened by bursting activity in the song system. This temporally registered signal functions as an aspect of the behavioral-goal memory and is sent to Av, where it can be directly compared to auditory feedback. Although speculative, an advantage of this model is that it may simplify learning by parsing different computations involved in performance evaluation across two separate and addressable sets of synaptic connections. Recall of the appropriate behavioral-goal memory is triggered on a moment-by-moment basis using corollary discharge signals within HVC. Evaluation of performance is accomplished by comparing this behavioral-goal memory to auditory feedback in Av. Another advantage of this model is that it does not invoke an auditory template in the $\mathrm{AC}$, instead it leans on recent functional data indicating the behavioral-goal memories are initially encoded in $\mathrm{HVC}$ and that connections from HVC back to the auditory system are essential for vocal imitation. Although it is unlikely that such a scenario can fully account for behavioral-goal memories, it can provide testable ideas for how motor circuits and memories can potentially engage with auditory circuits in the evaluation of performance.

\section{Conclusions}

Studies over the last several years have made considerable strides in identifying circuits for encoding behavioral-goal memories, but many questions still remain unanswered. Functional studies in the AC are needed to better understand its role in song learning. In addition, detailed studies aimed at dissecting how motor and auditory circuits are engaged to detect errors in performance are needed. Application of modern genetic, electrophysiological and imaging methods will be crucial to these efforts.

\section{Acknowledgments:}

The Roberts laboratory was supported by grants from the US National Institutes of Health (R01DC014364, R01NS108424, R01NS102488) and the National Science Foundation (IOS-1457206, IOS1451034) to T.F.R..

\section{References}

Adret P, Meliza CD, and Margoliash D. (2012). Song tutoring in presinging zebra finch juveniles biases a small population of higher-order song-selective neurons toward the tutor song. $\mathrm{J}$ Neurophysiol 108, 1977-1987. [PubMed: 22786956]

Akutagawa E, and Konishi M. (2010). New brain pathways found in the vocal control system of a songbird. Journal of Comparative Neurology 518, 3086-3100. [PubMed: 20533361] 
Bauer EE, Coleman MJ, Roberts TF, Roy A, Prather JF, and Mooney R. (2008). A synaptic basis for auditory-vocal integration in the songbird. J Neurosci 28, 1509-1522. [PubMed: 18256272]

Bolhuis JJ, and Gahr M. (2006). Neural mechanisms of birdsong memory. Nat Rev Neurosci 7, 347357. [PubMed: 16760915]

Bolhuis JJ, and Moorman S. (2015). Birdsong memory and the brain: in search of the template. Neurosci Biobehav Rev 50, 41-55. [PubMed: 25459663] * This paper provides an extensive review of the literature on immediate-early gene expression in the NCM and evidence for the presence of tutor song memory in the NCM.

Brainard MS, and Doupe AJ (2013). Translating birdsong: songbirds as a model for basic and applied medical research. Annu Rev Neurosci 36, 489-517. [PubMed: 23750515]

Canopoli A, Zai AT, and Hahnloser RHR (2016). Lesions of a higher auditory brain area during a sensorimotor period do not impair birdsong learning. Matters, 1-7.** Bilateral lesions of NCM after tutoring experience does not affect tutor song learning. This result suggests that NCM is not necessary for accessing the behavioral-goal memory used to guide song learning.

Canopoli A, Zai AT, and Hahnloser RHR (2017). Bilateral neurotoxic lesions in NCM before tutoring onset do not prevent succesful tutor song learning. Matters, 1-6.** Compelling evidence that NCM may not be necessary for storing behavioral goal memory. Bilateral lesions of NCM prior to tutoring experience did not prevent the pupil from copying the tutor song.

Cardin JA, Raksin JN, and Schmidt MF (2005). Sensorimotor nucleus NIf is necessary for auditory processing but not vocal motor output in the avian song system. J Neurophysiol 93, 2157-2166. [PubMed: 15590726]

Cardin JA, and Schmidt MF (2004). Auditory responses in multiple sensorimotor song system nuclei are co-modulated by behavioral state. J Neurophysiol 91, 2148-2163. [PubMed: 14724261]

Chen R, Puzerey PA, Roeser AC, Riccelli TE, Podury A, Maher K, Farhang AR, and Goldberg JH (2019). Songbird Ventral Pallidum Sends Diverse Performance Error Signals to Dopaminergic Midbrain. Neuron 103, 266-276 e264.

Coleman MJ, and Mooney R. (2004). Synaptic transformations underlying highly selective auditory representations of learned birdsong. J Neurosci 24, 7251-7265. [PubMed: 15317851]

Doupe AJ, and Kuhl PK (1999). Birdsong and human speech: common themes and mechanisms. Annu Rev Neurosci 22, 567-631. [PubMed: 10202549]

Fee MS, and Goldberg JH (2011). A hypothesis for basal ganglia-dependent reinforcement learning in the songbird. Neuroscience 198, 152-170. [PubMed: 22015923]

Gadagkar V, Puzerey PA, Chen R, Baird-Daniel E, Farhang AR, and Goldberg JH (2016). Dopamine neurons encode performance error in singing birds. Science 354, 1278-1282. [PubMed: 27940871]

Hahnloser RH, and Fee MS (2007). Sleep-related spike bursts in HVC are driven by the nucleus interface of the nidopallium. J Neurophysiol 97, 423-435. [PubMed: 17005618]

Hisey E, Kearney MG, and Mooney R. (2018). A common neural circuit mechanism for internally guided and externally reinforced forms of motor learning. Nat Neurosci 21, 589-597. [PubMed: 29483664]

Janata P, and Margoliash D. (1999). Gradual emergence of song selectivity in sensorimotor structures of the male zebra finch song system. Journal of Neuroscience 19, 5108-5118. [PubMed: 10366643]

Kearney MG, Warren TL, Hisey E, Qi J, and Mooney R. (2019). Discrete Evaluative and Premotor Circuits Enable Vocal Learning in Songbirds. Neuron.

Keller GB, and Hahnloser RH (2009). Neural processing of auditory feedback during vocal practice in a songbird. Nature 457, 187-190. [PubMed: 19005471]

Konishi M. (1965). The role of auditory feedback in the control of vocalization in the white-crowned sparrow. Zeitschrift fur Tierpsychologie 22, 770-783. [PubMed: 5874921]

Konopka G, and Roberts TF (2016). Animal Models of Speech and Vocal Communication Deficits Associated With Psychiatric Disorders. Biological psychiatry 79, 53-61. [PubMed: 26232298]

Kuhl PK, Williams KA, Lacerda F, Stevens KN, and Lindblom B. (1992). Linguistic experience alters phonetic perception in infants by 6 months of age. Science 255, 606-608. [PubMed: 1736364] 
Lewandowski B, Vyssotski A, Hahnloser RH, and Schmidt M. (2013). At the interface of the auditory and vocal motor systems: NIf and its role in vocal processing, production and learning. J Physiol Paris 107, 178-192. [PubMed: 23603062]

London SE, and Clayton DF (2008). Functional identification of sensory mechanisms required for developmental song learning. Nat Neurosci 11, 579-586. [PubMed: 18391944]

Mackevicius EL, and Fee MS (2018). Building a state space for song learning. Curr Opin Neurobiol 49, 59-68. [PubMed: 29268193] * The authors review the recent literature regarding the neuronal activity patterns in premotor, motor and auditory centers during song. They then generate a hypothesis of how reinforcement learning within and between auditory and premotor circuits may shape song timing in HVC and the production of the bird's adult song.

Mandelblat-Cerf Y, Las L, Denisenko N, and Fee MS (2014). A role for descending auditory cortical projections in songbird vocal learning. eLife 3.

Marler P. (1970). A comparative approach to vocal learning: song development in white-crowned sparrows. Journal of Comparative Physiological Psychology Monograph 71, 1-25.

McCasland JS (1987). Neuronal control of birdsong production. Journal of Neuroscience 7, 23-39. [PubMed: 3806194]

Mody M, and Belliveau JW (2013). Speech and Language Impairments in Autism: Insights from Behavior and Neuroimaging. N Am J Med Sci (Boston) 5, 157-161. [PubMed: 24349628]

Mooney R. (2009). Neural mechanisms for learned birdsong. Learn Mem 16, 655-669. [PubMed: 19850665]

Mooney R, and Prather JF (2005). The HVC microcircuit: the synaptic basis for interactions between song motor and vocal plasticity pathways. J Neurosci 25, 1952-1964. [PubMed: 15728835]

Moore JM, and Woolley SMN (2019). Emergent tuning for learned vocalizations in auditory cortex. Nat Neurosci 22, 1469-1476. [PubMed: 31406364] ** This paper shows evidence for higher auditory regions in the representation of vocalizations. Under normal rearing conditions, neurons in the higher auditory regions, including the NCM, are more sensitive to conspecific songs than heterospecific songs. However, when birds are cross reared with parents of another species, these neurons become more sensitive to their tutor bird's species' song.

Moorman S, Mello CV, and Bolhuis JJ (2011). From songs to synapses: molecular mechanisms of birdsong memory. Molecular mechanisms of auditory learning in songbirds involve immediate early genes, including zenk and arc, the ERK/MAPK pathway and synapsins. BioEssays : news and reviews in molecular, cellular and developmental biology 33, 377-385.

Nick TA, and Konishi M. (2005a). Neural auditory selectivity develops in parallel with song. J Neurobiol 62, 469-481. [PubMed: 15616963]

Nick TA, and Konishi M. (2005b). Neural song preference during vocal learning in the zebra finch depends on age and state. J Neurobiol 62, 231-242. [PubMed: 15459895]

Poremba A, Bigelow J, and Rossi B. (2013). Processing of communication sounds: contributions of learning, memory, and experience. Hear Res 305, 31-44. [PubMed: 23792078]

Portfors CV, and Perkel DJ (2014). The role of ultrasonic vocalizations in mouse communication. Curr Opin Neurobiol 28, 115-120. [PubMed: 25062471]

Prather JF, Peters S, Nowicki S, and Mooney R. (2010). Persistent representation of juvenile experience in the adult songbird brain. Journal of Neuroscience 30, 10586-10598. [PubMed: 20686001]

Roberts TF, Gobes SM, Murugan M, Olveczky BP, and Mooney R. (2012). Motor circuits are required to encode a sensory model for imitative learning. Nat Neurosci 15, 1454-1459. [PubMed: 22983208]

Roberts TF, Hisey E, Tanaka M, Kearney MG, Chattree G, Yang CF, Shah NM, and Mooney R. (2017). Identification of a motor-to-auditory pathway important for vocal learning. Nat Neurosci. 20(7):978-986. [PubMed: 28504672] ** This research identified population of HVC neurons projecting to the nucleus Avalanche, relaying a copy of the premotor song program to the auditory system. These neurons are proposed to participate to auditory feedback-based error signaling, as their ablation in young birds prevents them from developing a good copy of their tutors' song, while in adults it hinders the deafening-induced song degradation. 
Roberts TF, and Mooney R. (2013). Motor circuits help encode auditory memories of vocal models used to guide vocal learning. Hear Res 303, 48-57. [PubMed: 23353871]

Roberts TF, Tschida KA, Klein ME, and Mooney R. (2010). Rapid spine stabilization and synaptic enhancement at the onset of behavioural learning. Nature 463, 948-952. [PubMed: 20164928]

Ross MT, Flores D, Bertram R, Johnson F, Wu W, and Hyson RL (2019). Experience-Dependent Intrinsic Plasticity During Auditory Learning. J Neurosci 39, 1206-1221. [PubMed: 30541908] * This research identifies changes to the intrinsic physiological properties of HVC neurons following auditory experience with a song tutor, suggesting that learning may engage intrinsic plasticity within this song motor circuit.

Roy A, and Mooney R. (2009). Song decrystallization in adult zebra finches does not require the song nucleus NIf. J Neurophysiol 102, 979-991. [PubMed: 19515953]

Shank SS, and Margoliash D. (2009). Sleep and sensorimotor integration during early vocal learning in a songbird. Nature 458, 73-77. [PubMed: 19079238]

Solis MM, and Doupe AJ (1999). Contributions of tutor and bird's own song experience to neural selectivity in the songbird anterior forebrain. J Neurosci 19, 4559-4584. [PubMed: 10341255]

Solis MM, and Doupe AJ (2000). Compromised neural selectivity for song in birds with impaired sensorimotor learning. Neuron 25, 109-121. [PubMed: 10707976]

Sperdin HF, and Schaer M. (2016). Aberrant Development of Speech Processing in Young Children with Autism: New Insights from Neuroimaging Biomarkers. Frontiers in neuroscience 10, 393. [PubMed: 27610073]

Tager-Flusberg H, Paul R, and Lord CE (2005). Language and communication in autism In Handbook of Autism and Pervasive Developmental Disorders, and Behavior. Volkmar RPF, Klin A, \& Cohen DJ (Eds.), ed. (New York: : Wiley), pp. 335-364.

Tanaka M, Sun F, Li Y, and Mooney R. (2018). A mesocortical dopamine circuit enables the cultural transmission of vocal behaviour. Nature 563, 117-120. [PubMed: 30333629] ** Dopaminergic signaling in the tutee's HVC during tutoring experience is necessary for learning. Optogeneticallydriving dopaminergic afferents in HVC is sufficient to favor the memorization of a tutor's song played by a speaker, a normally non-permissive condition.

Vallentin D, Kosche G, Lipkind D, and Long MA (2016). Neural circuits. Inhibition protects acquired song segments during vocal learning in zebra finches. Science 351, 267-271. [PubMed: 26816377] ** This study finds that premotor neurons in HVC of juvenile birds are responsive to playback of their tutors song and that vocal imitation is associated with increased inhibition onto premotor neurons that functions to suppress these auditory responses.

Volman SF (1993). Development of neural selectivity for birdsong during vocal learning. Journal of Neuroscience 13, 4737-4747. [PubMed: 8229196]

Werker JF, and Tees RC (1992). The organization and reorganization of human speech perception. Annu Rev Neurosci 15, 377-402. [PubMed: 1575448]

Woolley SM, Fremouw TE, Hsu A, and Theunissen FE (2005). Tuning for spectro-temporal modulations as a mechanism for auditory discrimination of natural sounds. Nat Neurosci 8, 13711379. [PubMed: 16136039]

Xiao L, Chattree G, Oscos FG, Cao M, Wanat MJ, and Roberts TF (2018). A Basal Ganglia Circuit Sufficient to Guide Birdsong Learning. Neuron 98, 208-221 e205.

Yanagihara S, and Yazaki-Sugiyama Y. (2016). Auditory experience-dependent cortical circuit shaping for memory formation in bird song learning. Nature communications 7, 11946.* In this paper, authors find tutor song-selective neurons in NCM after juveniles go through a tutoring experience. The authors interpret this as evidence for tutor song memory located in NCM. While this could be a perceptual memory of the tutor song, no functional experiments were included to support that these memories are involved in guiding song learning.

Zhao W, Garcia-Oscos F, Dinh D, and Roberts TF (2019). Inception of memories that guide vocal learning in the songbird. Science 366, 83-89. [PubMed: 31604306] ** Optogenetic activation of NIf projections to HVC in young birds was used to implant memories for song syllable durations used to guide vocal learning. Optical activation of this pathway can override normal tutoring experience and genetic lesions of NIf neurons projecting to HVC block a young bird's ability to learn from a song tutor. 


\section{Highlights}

- Memories formed during social experience are used to guide vocal imitation

- $\quad$ Recent functional studies indicate that vocal motor circuits play an essential role in the initial encoding of memories used to guide vocal imitation

- Lesion studies question the role of auditory circuits in storing memories used to guide vocal imitation

- $\quad$ Studies implanting memories for song syllable duration and the role of dopamine in facilitating song memory formation are reviewed

- Corollary discharge pathways in premotor circuits are hypothesized to directly engage song memories to allow evaluation of motor performance 
A

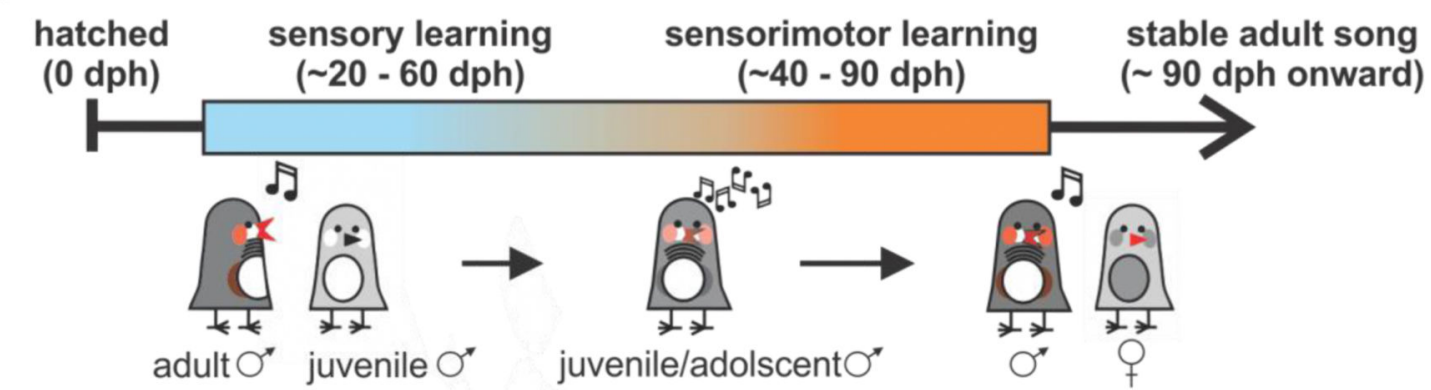

memorize temporal and practice song to use song to attract spectral properties of tutor song imitate tutor mate

B
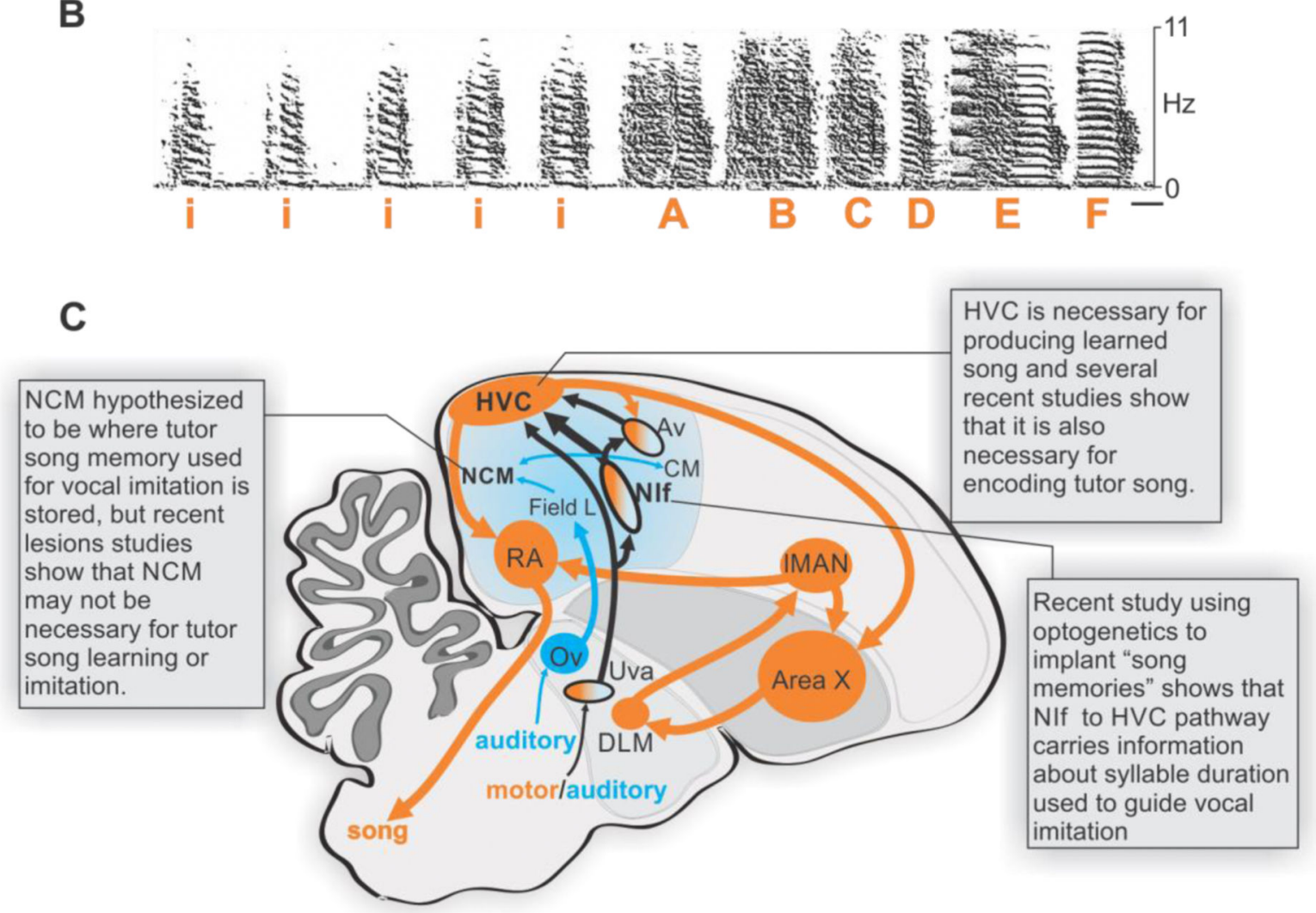

Figure 1 |. Overview of song learning and neural circuits for song [adapted from (Zhao et al., 2019)].

(A) Timeline for song learning in juvenile male zebra finches. Days post-hatching (dph). (B) Spectrogram of an adult zebra finch song illustrating introductory notes (i) and individual elements in the song ('A-F'). Scale bar, 50ms. (C) Parasagittal schematic of auditory (blue) and song motor circuits (orange). The striato-pallidal basal ganglia nucleus Area X, nucleus avalanche (Av), caudal mesopallium (CM), dorsolateral thalamic nucleus (DLM), primary auditory forebrain (field L), premotor song nucleus HVC (proper name), lateral magnocellular nucleus of the anterior nidopallium (LMAN), caudomedial nidopallium 
(NCM), nucleus interface of the nidopallium (NIf), thalamic nucleus ovoidalis (Ov), nucleus Uvaeformis (Uva), robust nucleus of the arcopallium (RA). 
a

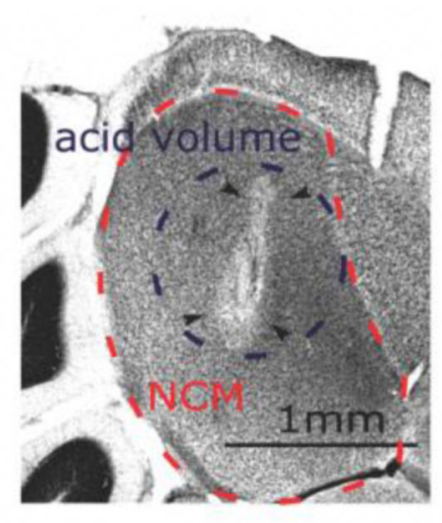

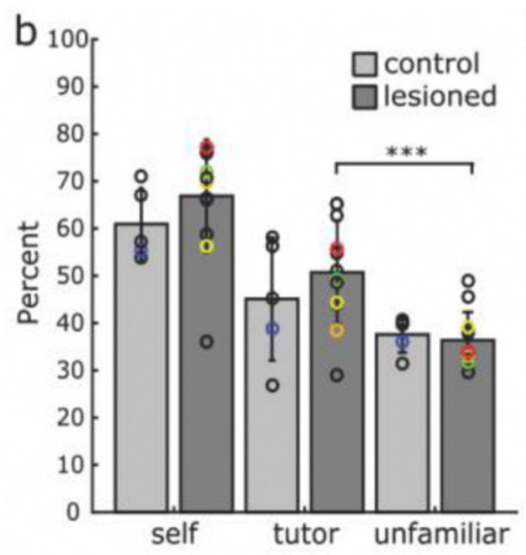

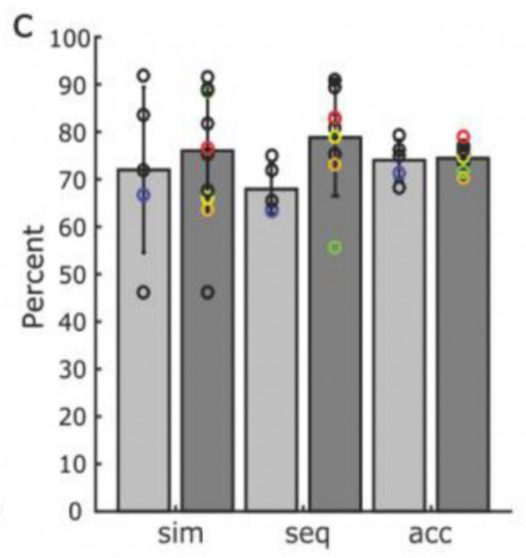

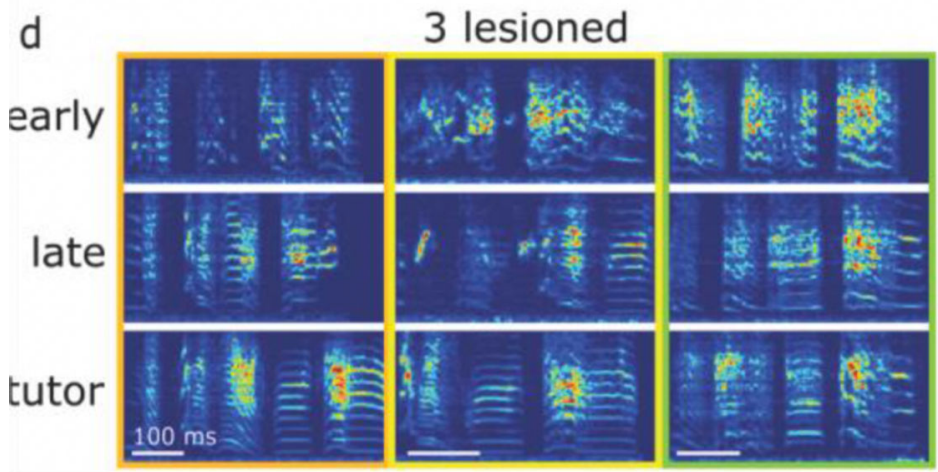
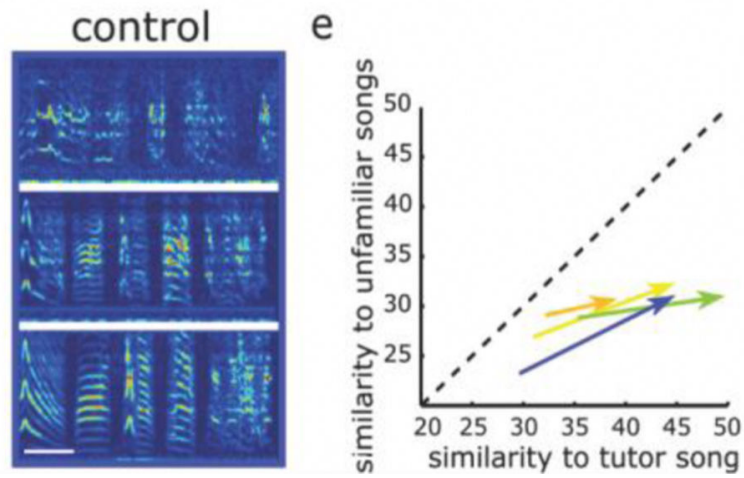

Figure 2 |. Bilateral neurotoxic lesions in NCM before tutoring onset do not prevent successful tutor song learning [reprinted from (Canopoli et al., 2017)].

(A) Nissl-stained brain section illustrating lesion to NCM. Birds lesioned as juveniles prior to tutoring. This image is from an adult illustrating amount of lesion remaining into adulthood. (B) Average similarity scores of juvenile song motifs with themselves (self), with tutor motifs (tutor), and with motifs from unfamiliar birds (unfamiliar). The bars show the mean \pm 1 standard deviation of all birds and the circles indicate the scores of individual birds. The scores for the NCM-lesioned bird shown in panel 'a' are highlighted in red and the four birds shown in panel 'd' are marked with according colors. The 3 similarity scores are each indistinguishable between NCM-lesioned birds (dark grey bars) and untreated control birds (light grey bars), revealing that NCM-lesioned birds are not impaired in their ability to memorize and subsequently learn a song. The 3 asterisks indicate that the average similarity score between lesioned juveniles and their tutor is significantly larger than the average similarity score between the lesioned juveniles and unfamiliar adult birds, showing that NCM-lesioned juveniles specifically imitated their tutor. (C) The song motifs of NCMlesioned birds and control birds at $75 \mathrm{dph}$ exhibit roughly identical percent similarity, sequential match, and accuracy in relation to tutor song. (D) Sample song-motif spectrograms from 3 birds with NCM lesions and from one control bird (right). The motifs shown on top ('early') were recorded at $50 \mathrm{dph}$, after 7 consecutive days of tutoring. The motifs in the middle ('late') were recorded at $75 \mathrm{dph}$, after 25 days of isolation from the tutor. Despite the NCM lesion and the isolation, the 'late' motifs in NCM-lesioned birds strongly resemble the tutors' motifs (bottom, 'tutor'). (E) The song changes in the 3 NCM- 
lesioned birds and the control bird in 'd' occurring between $50 \mathrm{dph}$ and $75 \mathrm{dph}$ (arrows) point more towards tutor song than towards unfamiliar song, demonstrating that within a week after NCM lesions, birds can memorize a tutor's song and subsequently learn to imitate it. 
A optogenetic tutoring

hatched ChR2 injection optical stimulation

(0 dph)

( $\sim 5 \mathrm{dph})$

(5 days)

analysis of adult song (90-120 dph)

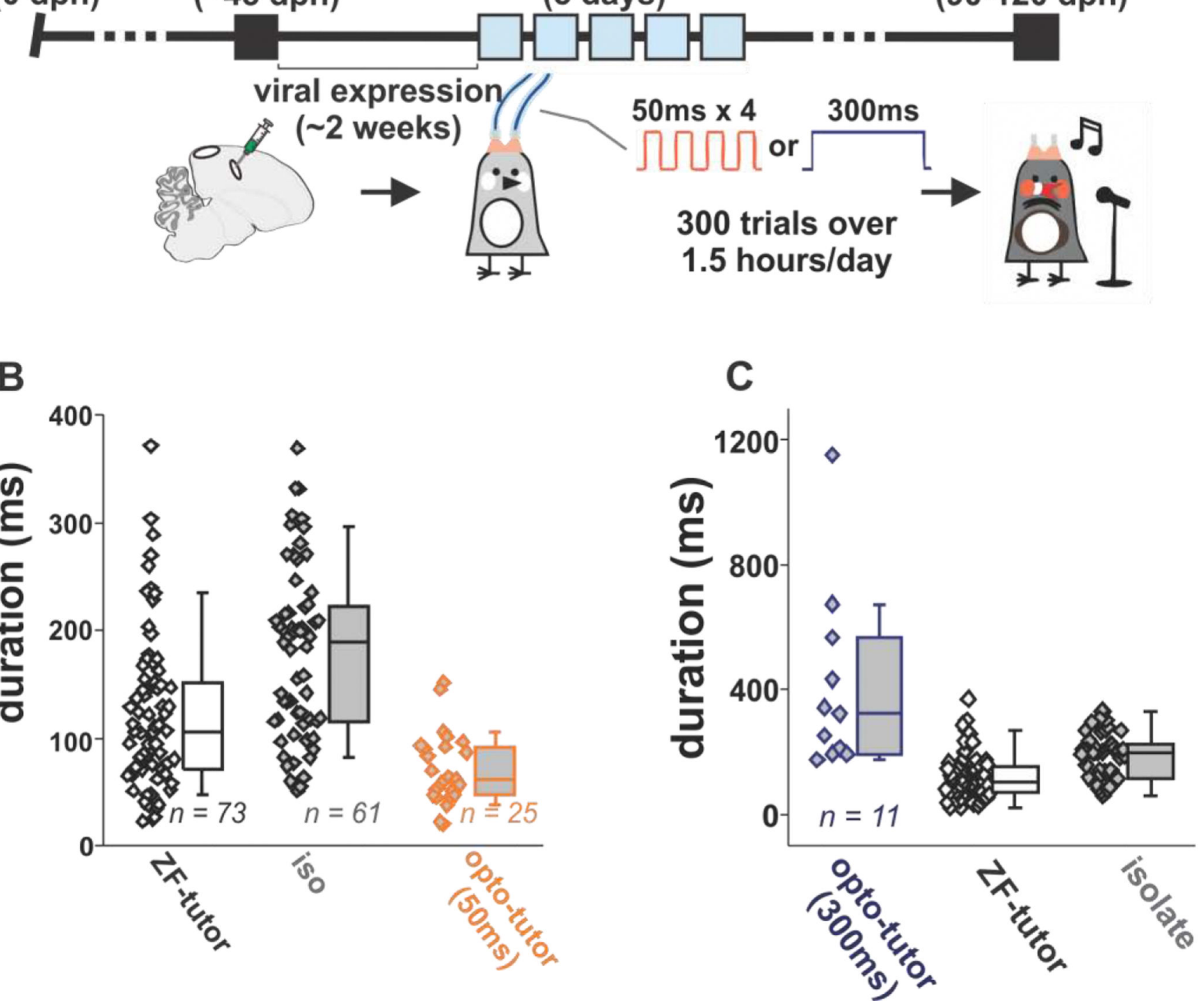

Figure 3 |. Optogenetic tutoring affects learning of song temporal structure [adapted from Zhao et al, 2019].

(A) Timeline for optogenetic tutoring experiments using 50-ms or 300-ms light pulses. (B) Song element duration of birds tutored by a zebra finch tutor (ZF-tutor, $n=73$ elements, 15 birds), isolate birds (iso, $\mathrm{n}=61$ elements, 10 birds), and birds 50-ms opto-tutored birds (opto-tutor, $\mathrm{n}=25$ elements, 7 birds). Mann-Whitney U tests: ZF-tutor birds versus isolate birds (by song element duration, $P=2.83 \times 10^{-5}$; by bird, $P=0.0025$ ); ZF-tutor birds versus 50-ms opto-tutored birds (by song element duration, $P=3.86 \times 10^{-4}$; by bird, $P=0.0031$ ); isolate birds versus $50-\mathrm{ms}$ opto-tutored birds (by song element duration, $P=4.67 \times 10^{-9}$; by bird, $\left.P=2.06 \times 10^{-4}\right)$. (C) Song element durations for birds opto-tutored with 300 -ms pulses 
(blue, gray fill) and comparisons with song element durations from normally reared (black, no fill) and isolate birds (black, gray fill). Mann-Whitney U tests: 300-ms opto-tutored birds versus isolate birds (by song element duration, $P=0.0010$; by bird, $P=2.0568 \times 10^{-4}$ ); 300ms opto-tutored birds versus ZF-tutored birds (by song element duration, $P=3.2549 \times 10^{-6}$; by bird, $P=2.4684 \times 10^{-4}$ ). 


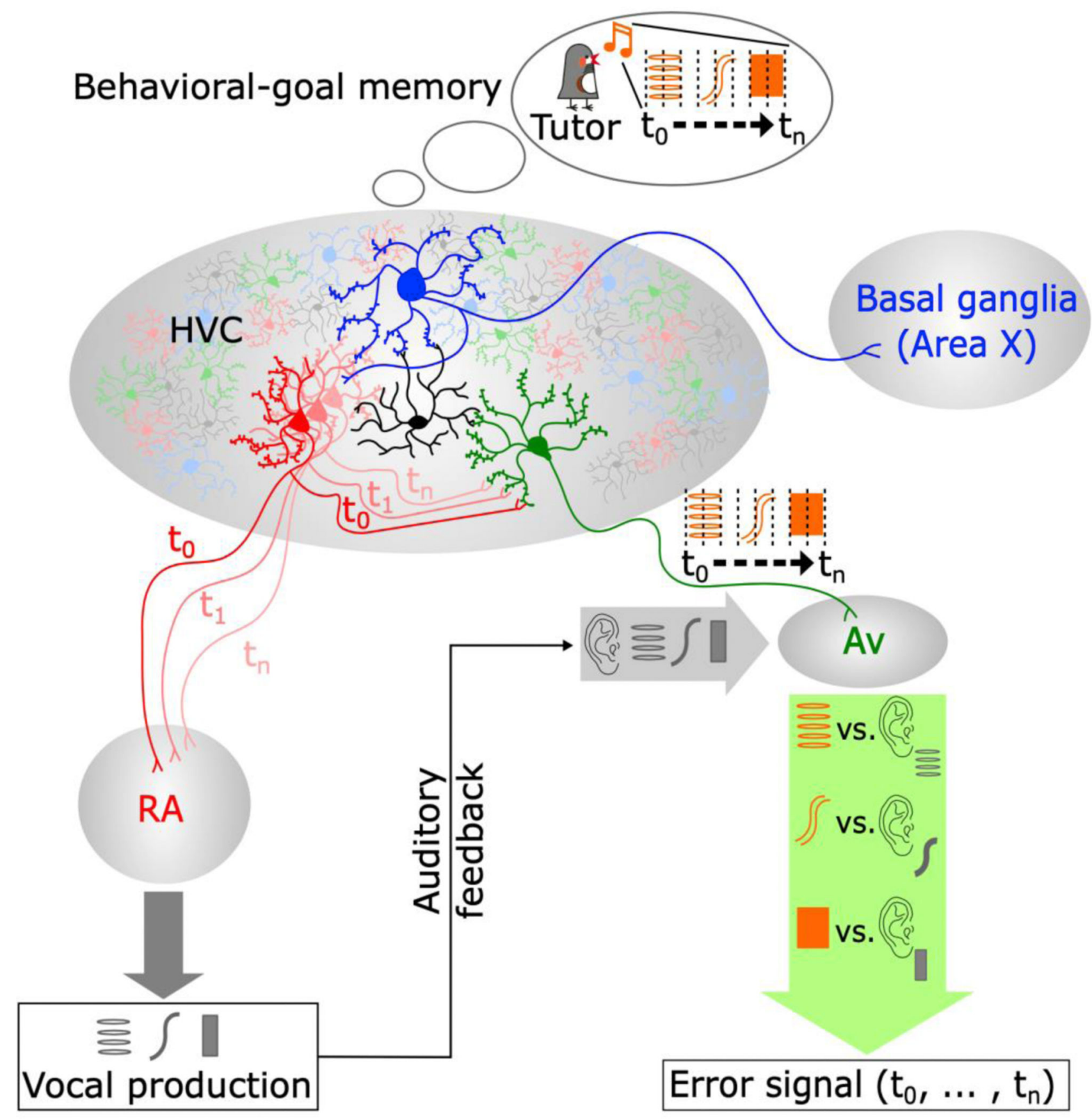

Figure $4 \mid$. Hypothesized model for aligning behavioral-goal memories and descending motor commands for song evaluation.

In this model, the behavioral-goal memory of the tutor song is represented in motor coordinates within HVC and is used to guide vocal learning. The known HVC neuronal subpopulations are represented (black, interneurons; blue, $\mathrm{HVC}_{\mathrm{X}}$; red, $\mathrm{HVC}_{\mathrm{RA}}$; green, $\mathrm{HVC}_{\mathrm{Av}}$ ). Connections to the end target nuclei and/or to HVC neuronal subpopulations are indicated by bifurcated axonal aspiny terminations onto the brain nuclei (or on the dendritic trees of the target neurons) (Mooney and Prather, 2005; Roberts et al., 2017). In this model, each $\mathrm{HVC}_{\mathrm{RA}}$ neurons conveys to RA information about a specific moment in song $\left(\mathrm{t}_{0-n}\right)$. A 
copy of this signal is used locally in $\mathrm{HVC}$, via local axonal collaterals of $\mathrm{HVC}_{\mathrm{RA}}$ neurons, to recall the behavioral-goal memory for each moment in the song. Signaling through $\mathrm{HVC}_{\mathrm{Av}}$ neurons, a temporally registered predictive signal that represents the behavioral-goal memory for each time-step in the song is relayed to Avalanche. Once in the auditory system, this behavioral-goal memory is compared with the auditory feedback to evaluate song performance. 